University of Nebraska - Lincoln

DigitalCommons@University of Nebraska - Lincoln

$5-1-2000$

\title{
Magnetic spectroscopy and characterization of $\mathrm{La}_{0.65} \mathrm{~Pb}_{0.35} \mathrm{MnO}_{3}$
}

\author{
M. I. Chipara \\ University of Nebraska-Lincoln, mchipara@utpa.edu \\ Shireen Adenwalla \\ University of Nebraska-Lincoln, sadenwalla1@unl.edu \\ Peter A. Dowben \\ University of Nebraska-Lincoln, pdowben@unl.edu \\ Qin Lan Xu \\ University of Nebraska-Lincoln \\ Sy_Hwang Liou \\ University of Nebraska-Lincoln, sliou@unl.edu \\ See next page for additional authors
}

Follow this and additional works at: https://digitalcommons.unl.edu/physicsdowben

Part of the Physics Commons

Chipara, M. I.; Adenwalla, Shireen; Dowben, Peter A.; Xu, Qin Lan; Liou, Sy_Hwang; and Shoemaker, R., "Magnetic spectroscopy and characterization of $\mathrm{La}_{0.65} \mathrm{~Pb}_{0.35} \mathrm{MnO}_{3}$ " (2000). Peter Dowben Publications. 31.

https://digitalcommons.unl.edu/physicsdowben/31

This Article is brought to you for free and open access by the Research Papers in Physics and Astronomy at DigitalCommons@University of Nebraska - Lincoln. It has been accepted for inclusion in Peter Dowben Publications by an authorized administrator of DigitalCommons@University of Nebraska - Lincoln. 


\section{Authors}

M. I. Chipara, Shireen Adenwalla, Peter A. Dowben, Qin Lan Xu, Sy_Hwang Liou, and R. Shoemaker 


\title{
Magnetic spectroscopy and characterization of $\mathrm{La}_{0.65} \mathrm{~Pb}_{0.35} \mathrm{MnO}_{3}$
}

\author{
M. I. Chipara, S. Adenwalla, ${ }^{\text {a) }}$ P. A. Dowben, Qin Lan Xu, Sy-Hwang Liou, \\ and R. Shoemaker \\ Department of Physics and Astronomy and the Center for Materials Research and Analysis, Behlen \\ Laboratory of Physics, University of Nebraska, Lincoln, Nebraska 68500-0111
}

\begin{abstract}
Crystalline films of $\mathrm{La}_{0.65} \mathrm{~Pb}_{0.35} \mathrm{MnO}_{3}$ grown on $\mathrm{Si}$ (100) substrates by rf sputtering have been investigated by ferromagnetic resonance and MOKE. The dependence of the ferromagnetic resonance line parameters on the sample orientation and temperature is investigated. An expression for the angular dependence of the resonance field on the orientation of the sample is proposed, based on the assumption that close to the Curie temperature the Zeeman and demagnetizing terms are dominant. MOKE data support this hypothesis. The temperature dependence of the magnetization at saturation, in relative units, estimated from ferromagnetic resonance agrees with SQUID data. (C) 2000 American Institute of Physics. [S0021-8979(00)67008-1]
\end{abstract}

\section{INTRODUCTION}

In recent years there has been a dramatic rebirth of interest in transition metal oxides, and many intriguing phenomena have been discovered. ${ }^{1,2}$ The driving force behind these phenomena lies in the coupling between charge, spin and lattice degrees of freedom.

Most studies on perovskites using ferromagnetic resonance (FMR) were performed on powders. ${ }^{1,3-6}$ FMR investigations on thin films of perovskites were usually restricted to the parallel and perpendicular orientations. The aim of this contribution is a detailed study concerning the angular dependence of FMR spectra on a crystalline epitaxial sample of $\mathrm{La}_{x} \mathrm{~Pb}_{1-x} \mathrm{MnO}_{3}$ perovskite.

\section{EXPERIMENT}

Crystalline films of $\mathrm{La}_{0.65} \mathrm{~Pb}_{0.35} \mathrm{MnO}_{3}$ were grown on $\mathrm{Si}$ (100) by rf sputtering in a 4:1 argon/oxygen atmosphere maintained at 15 mTorr. The bulk chemical composition of the films was determined from energy dispersive analysis of $\mathrm{x}$-ray emission spectroscopy. The films were subsequently annealed at $873 \mathrm{~K}$ in an oxygen atmosphere of 2 atm at 1173 $\mathrm{K}$ for $5 \mathrm{~h}$ in order to improve the compositional homogeneity of the samples. The thickness of the $\mathrm{La}_{0.65} \mathrm{~Pb}_{0.35} \mathrm{MnO}_{3}$ sample is nominally $1 \mu \mathrm{m}$.

The samples were studied by FMR using a Bruker D-200 spectrometer operating in the $X$ band $(\approx 9 \mathrm{GHz})$. The dependence of resonance line parameters (line position, shape and width) on the angle between the external field and the plane of the sample as well the temperature dependence was studied. The magnetic hysteresis loops were measured at room temperature by MOKE.

\section{RESULTS AND DISCUSSION}

The experimental data indicate a strong dependence of the resonance line position and width on the orientation of the film. The external magnetic field is applied at an angle

\footnotetext{
a) Author to whom correspondence should be addressed, electronic mail: sadenwal@unlinfo.unl.edu; Fax: 402-472-2879
}

$\theta_{H}$ with the normal to the plane of the sample ([100] and $[010])$ and $\theta$ represents the angle between the magnetization of the sample and the normal to the plane of the film. In the absence of the external magnetic field or if $\theta_{H}=90^{\circ}$, the magnetization lies in the plane of the sample $\left(\theta=90^{\circ}\right)$. The dependence of FMR spectrum on the angle between the external magnetic field $\mathbf{H}$ and the plane of the sample, is represented in Fig. 1. The position of the FMR line is shifted towards higher external magnetic fields as the orientation of the sample is changed from the in-plane configuration $\left(\theta_{H}\right.$ $\left.=90^{\circ}\right)$ to the out-of-plane configuration $\left(\theta_{H}=0^{\circ}\right)$. A second strong and narrow resonance has been noticed only for $\theta_{H}$ close to zero. ${ }^{7}$ We ascribe this line to magnetostatic (standing spin wave resonance) modes. The FMR spectrum and the first magnetostatic modes were recorded only close to the Curie temperature as our spectrometer is limited to magnetic fields of about $1 \mathrm{~T}$.

The following terms contribute to the free energy, $E$, of a ferromagnetic sample, ${ }^{3-12}$

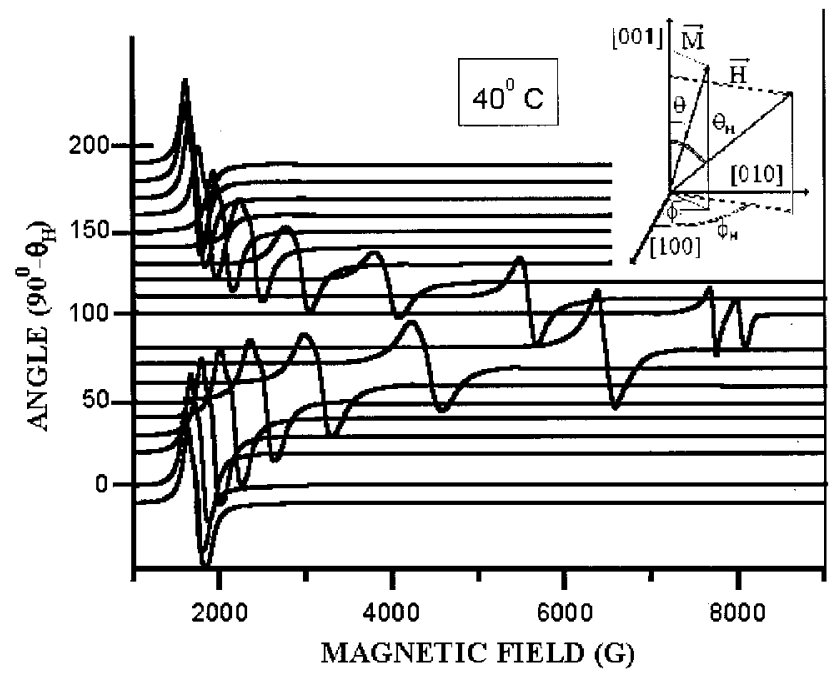

FIG. 1. FMR spectra at various angles between the external magnetic field and the normal to the plane of the sample $(T=313 \mathrm{~K})$. The orientation of the magnetic field and magnetization is given in the inset. 


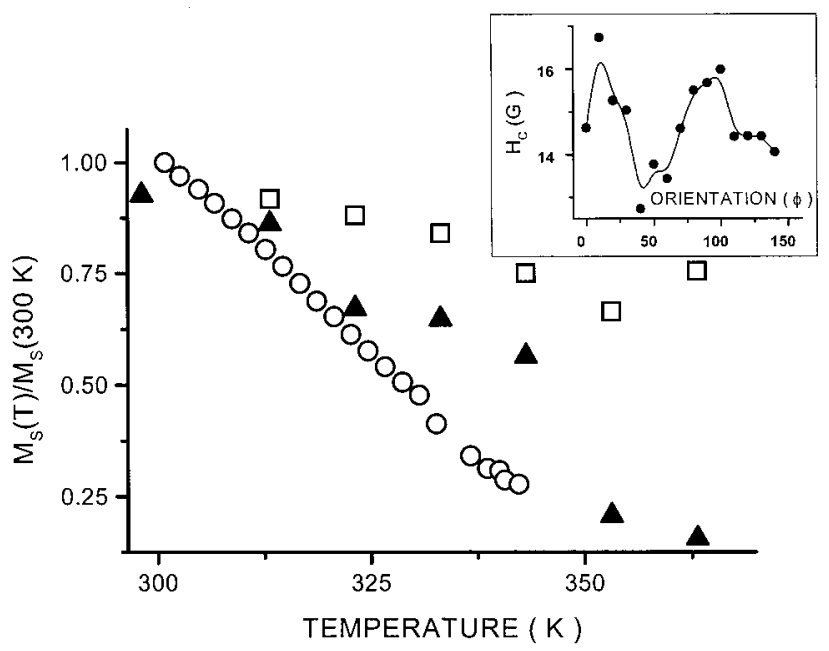

FIG. 2. The temperature dependence of the relative magnetization as measured by SQUID and derived from FMR data using relations (4) and (5), respectively. $(\bigcirc)$ represents the relative magnetization, as measured by SQUID, ( $\square$ ) is the relative magnetization, estimated from FMR data using Eq. (4), and $(\mathbf{\Delta})$ represents the relative magnetization, obtained from FMR data using Eq. (5). The inset shows the angular dependence of the coercive field, derived from the hysteresis curves, by MOKE measurements (in-plane configuration). The line is only a guide to the eye.

$$
\begin{aligned}
E= & -\mathbf{M H}+K_{1}\left(\alpha_{[100]}^{2} \alpha_{[010]}^{2}+\alpha_{[100]}^{2} \alpha_{[001]}^{2}+\alpha_{[010]}^{2} \alpha_{[001]}^{2}\right) \\
& +K_{2}\left(\alpha_{[100]}^{2} \alpha_{[101]}^{2} \alpha_{[001]}^{2}\right)+\left(N_{[001]} M_{[001]}^{2}\right. \\
& \left.+N_{[010]} M_{[010]}^{2}+N_{[100]} M_{[100]}^{2}\right)+\cdots .
\end{aligned}
$$

The first term is the Zeeman energy, the next two reflect the contribution of the cubic magnetocrystalline anisotropy and the last is associated with demagnetizing effects. $M$ is the magnetization, $H$ is the intensity of the applied magnetic field, $K_{1}$ and $K_{2}$ are the first and, respectively, the second order cubic magnetocrystalline anisotropy constants, and $\alpha_{i}$ $(i=[100],[010],[001])$ are the direction cosines of the magnetization with respect to the coordinate axes (Fig. 1). In the derivation of (1), only the diagonal components of the demagnetizing tensor $\mathbf{N} \quad\left(N_{i i}=N_{i}\right.$, where $i=[100]$, [010],[001]) were considered. We have chosen the $O Z$ axis along the growth direction of the film (along the [001] axis).

The position of the resonance line is estimated using ${ }^{3-12}$

$$
\begin{aligned}
& \left(\frac{\omega}{\gamma}\right)^{2}=H_{\mathrm{eff}}^{2}=\frac{1}{M^{2} \sin ^{2} \theta_{0}}\left[\left(\frac{\partial^{2} E}{\partial \theta^{2}}\right)\left(\frac{\partial^{2} E}{\partial \varphi^{2}}\right)-\left(\frac{\partial^{2} E}{\partial \theta \theta \varphi}\right)^{2}\right], \\
& \left.\left(\frac{\partial E}{\partial \theta}\right)\right|_{\theta=\theta_{0}}=0,\left.\quad\left(\frac{\partial E}{\partial \varphi}\right)\right|_{\varphi=\varphi_{0}}=0
\end{aligned}
$$

The derivatives of the free energy are estimated at equilibrium $\left(\theta=\theta_{0}, \varphi=\varphi_{0}\right)$. In our case, $M$ is equal to the magnetization at saturation, $M_{S}$, as the coercive field at room temperature is of the order of $10^{2} \mathrm{G}$ and the lowest resonance field is above $2000 \mathrm{G}$. The in-plane magnetic hysteresis loops obtained by MOKE indicate that the effect of the inplane magnetocrystalline anisotropy at room temperature, is smaller than $10^{2} \mathrm{G}$ (see the inset of Fig. 2). Hence this contribution to the FMR spectrum is negligible. From the posi- tion of the resonance line, the effective magnetization and the effective field $H_{\text {eff }}$ may be estimated, ${ }^{10-15}$

$$
\begin{aligned}
& \left.\left(\frac{\omega}{\gamma}\right)^{2}\right|_{\theta=0}=\left(H_{\mathrm{eff}}^{\perp}\right)^{2}=\left(H_{\perp}-4 \pi M_{\mathrm{eff}}\right)^{2}, \\
& \left.\left(\frac{\omega}{\gamma}\right)^{2}\right|_{\theta=\pi / 2}=\left(H_{\mathrm{eff}}^{\|}\right)^{2}=H_{\|}\left(H_{\|}+4 \pi M_{\mathrm{eff}}\right),
\end{aligned}
$$

where $H_{\perp}$ and $H_{\|}$are the resonance field for the out-of-plane and in-plane configurations, respectively, $M_{\text {eff }}$ is an effective magnetization $\left(M_{\mathrm{eff}}=M_{S}-2 K_{1} /\left(4 \pi M_{S}\right)\right)$ which may contain a contribution from the magnetocrystalline anisotropy. This equation, initially developed for Co films, ${ }^{13}$ has been derived supposing a uniaxial perpendicular anisotropy. The equation has been used in the discussion of FMR data on $\mathrm{La}_{0.67} \mathrm{Ba}_{0.33} \mathrm{MnO}_{z} \cdot{ }^{14}$ For $H_{\text {eff }}^{\perp}=H_{\text {eff }}^{\|},{ }^{13,14}$ taking into account that $\gamma=2 \pi g \mu_{B} / h,{ }^{14}$ where $\mu_{B}$ is the electronic Bohr magneton and $h$ is the Planck constant, Eq. (4) allows the estimation of the $M_{\text {eff }}$ and of the effective $g$ value, $g_{\text {eff }}$.

The relation between the $M_{\text {eff }}$, as estimated from FMR using Eq. (4), and $M_{S}$, as measured by SQUID, has been analyzed for $\mathrm{La}_{0.67} \mathrm{Ba}_{0.33} \mathrm{MnO}_{z} \cdot{ }^{14}$ Using the same approach, we represented in Fig. 2, the temperature dependence of the relative magnetization (at saturation and effective, respectively). The agreement between the data calculated from FMR data and the data obtained by SQUID is not very good. The result is explained by the discrepancy between $M_{S}$ and $M_{\text {eff }}$, i.e., $M_{\text {eff }}-M_{S}$ and is due to the term $2 K_{1} / M_{S}$ which contains the effect of both the demagnetizing term and the magnetocrystalline anisotropy perpendicular to the plane of the film. For thin films of $\mathrm{La}_{0.67} \mathrm{Ba}_{0.33} \mathrm{MnO}_{z}$, Lofland et al. ${ }^{14}$ reported that the temperature dependence of $K_{1}$, is not monotonous, presenting an extremum below Curie temperature. They ascribed ${ }^{14}$ this behavior to magnetostriction. Hence we conclude that in our samples the strains are not negligibles, as there is a lattice mismatch between the film and the substrate. Using Eq. (4) we were not able to obtain reasonable values for $H_{\text {eff }}$, which is expected to be around $3500 \mathrm{G}$ (close to $g=2.00$ ) above $360 \mathrm{~K}$, as the sample undergoes a transition from ferromagnetism to paramagnetism. This is expected as in Eq. (4), $M_{\text {eff }} \neq M_{S}$.

We postulate that the dependence of the FMR spectrum on the angle $\theta_{H}$ denoted as the out-of-plane angular dependence) is dominated by the Zeeman and shape (demagnetizing) terms. For ferromagnetic thin films, the dominant contribution to the demagnetizing field is due to $N_{[001]}$. However, this term may include an axial out-of-plane contribution due to the magnetocrystalline anisotropy. Under these circumstances, the equilibrium orientation of the magnetization corresponds to $\varphi_{0}=\varphi_{H}$, as the in-plane anisotropy is neglected.

For the out-of-plane orientation, a slight misalignment between the magnetic field and sample's magnetization has been introduced supposing $\theta_{0}=\theta_{H}+\varepsilon$. Based on the results obtained by MOKE, which indicate that the magnetocrystalline anisotropy is of the order of $10^{1}-10^{2} \mathrm{G}$, and taking into account that within the ferromagnetic to paramagnetic transition range the contribution of the magnetization at satura- 


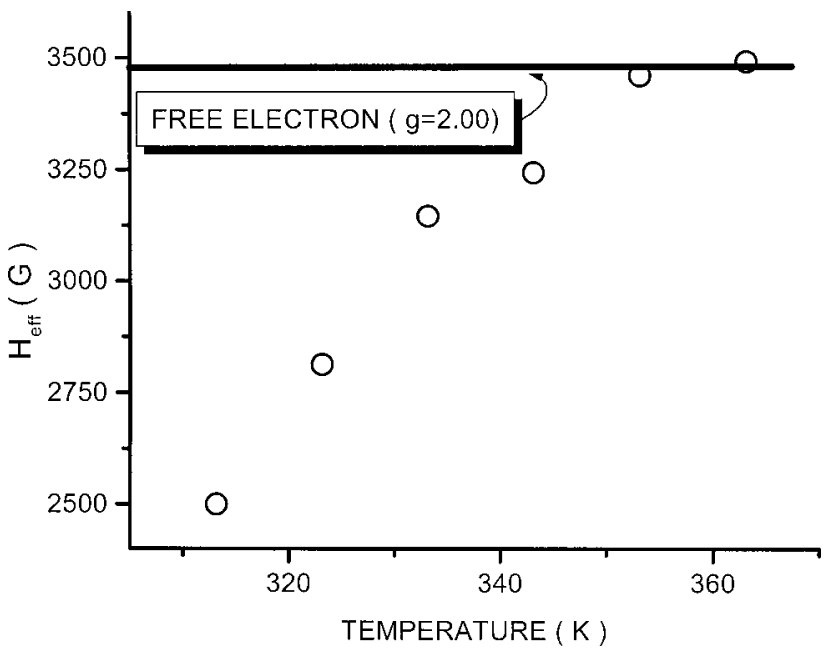

FIG. 3. The temperature dependence of the effective field, estimated from FMR data, using Eq. (5).

tion is small in comparison to the external magnetic field (which is larger than $2000 \mathrm{G}$ ), we concluded that $\epsilon$ is very small. Hence, we have found the following expressions for $H_{\|}$and $H_{\perp}$,

$$
\begin{aligned}
& \left.\left(\frac{\omega}{\gamma}\right)^{2}\right|_{\theta=\pi / 2}=\left(H_{\mathrm{eff}}^{\|}\right)^{2}=H\left(H+2 N_{[001]} M\right), \\
& H_{\|}=\sqrt{N_{[001]}^{2} M^{2}+H_{\mathrm{eff}}^{2}}-N_{[001]} M, \\
& \left.\left(\frac{\omega}{\gamma}\right)^{2}\right|_{\theta=0}=\left(H_{\mathrm{eff}}^{\perp}\right)^{2}=H\left(H-2 N_{[001]} M\right), \\
& H_{\perp}=\sqrt{N_{[001]}^{2} M^{2}+H_{\mathrm{eff}}^{2}}+N_{[001]} M .
\end{aligned}
$$

As may be noticed from Fig. 2, the temperature dependence of the relative magnetization at saturation is better described using Eq. (5) than Eq. (4).$^{10-14}$ In the derivation of (5) we have neglected all magnetocrystalline effects, both in-plane and out-of-plane. This implies that $\phi_{0}=\phi_{H}$, i.e., the in-plane component of the magnetization is parallel to the in-plane component of the external magnetic field and allows us to calculate $M_{S}$ directly.

The approximation used by us claims that the deviations in the orientation of the magnetization relative to the external magnetic fields are very small. It leads to an acceptable temperature dependence of the effective field, which is monotonically increased towards $H_{\text {eff }}=3500 \mathrm{G}$ (Fig. 3) as the temperature of the sample is raised towards the Curie temperature. This corresponds to a decrease of the $g$ factor from a value close to 4 (at room temperature) up to $g$ $\approx 2.00$ around $360 \mathrm{~K}$. This behavior reflects the transition from ferromagnetism to paramagnetism.

\section{CONCLUSIONS}

FMR and MOKE spectrometry investigations on $\mathrm{La}_{0.65} \mathrm{~Pb}_{0.35} \mathrm{MnO}_{3}$ are reported. An expression for the posi- tion of the resonance field corresponding to the in-plane and out-of-plane configuration is proposed, postulating that the Zeeman and demagnetizing terms govern the evolution of the magnetization, and supposing that the misalignment of the magnetization relative to the external magnetic field is very small. It is suggested that these equations may be used in the discussion of FMR data on soft magnetic films. The magnetic hysteresis curves obtained by MOKE spectroscopy indicate that the in-plane magnetocrystalline anisotropy is small, supporting the above-mentioned hypothesis. From the resonance fields corresponding to the in-plane and out-ofplane configurations, we have estimated the magnetization at saturation and the effective magnetic field. The temperature dependence of the magnetization, in relative units, is in good agreement with the data obtained by magnetic measurements (SQUID). The small discrepancies may be due to the fact that we have neglected all magnetocrystalline anisotropy and the magnetostriction contributions. The temperature dependence of the effective field is compatible with a Curie temperature of about $360 \mathrm{~K}$.

\section{ACKNOWLEDGMENTS}

This work was supported by the NSF through Grant Nos. DMR-98-06308, DMR 98-02126, and by the Center for Materials Research and Analysis (CMRA) at the University of Nebraska.

${ }^{1}$ J. M. D. Coey and M. Virett, Adv. Phys. 48, 167 (1999).

${ }^{2}$ M. Imada, A. Fujimori, and Y. Tokura, Rev. Mod. Phys. 70, 1039 (1998).

${ }^{3}$ C. Searle and S. T. Wang, Can. J. Phys. 47, 2703 (1969).

${ }^{4}$ G. Srinivasan, V. Buresh Babu, and M. Seehra, Appl. Phys. Lett. 67, 2090 (1995).

${ }^{5}$ M. T. Causa et al., Phys. Rev. B 58, 3233 (1998).

${ }^{6}$ F. Rivadulla, L. E. Hueso, C. Jardon, C. Vazquez-Vazquez, M. A. LopezQuintela, J. Rivas, M. T. Causa, C. A. Ramos, and R. D. Sanchez, J. Magn. Magn. Mater. 196-197, 470 (1999).

${ }^{7}$ S. E. Lofland, S. M. Bhagat, C. Kwon, M. C. Robson, R. P. Sharma, R. Ramesh, and T. Venkatesan, Phys. Lett. A 209, 246 (1995).

${ }^{8}$ Ferromagnetic Resonance, edited by S. V. Vonsovskii (Pergamon, Oxford, 1966), p. 22.

${ }^{9}$ S. M. Rezende, J. A. S. Moura, F. M. De Aguiar, and W. Schreiner, Phys. Rev. B 49, 15105 (1994).

${ }^{10}$ J. Du, J. Wu, L. N. Tong, M. Lu, J. H. Du, M. H. Pan, H. R. Zhai, and H. Xia, Phys. Status Solidi A 167, 183 (1998).

${ }^{11}$ L. N. Tong, J. Du, M. Lu, X. S. Wu, K. Xia, H. W. Zhao, B. You, H. R. Zhai, and H. Xia, Phys. Status Solidi A 165, 261 (1998).

${ }^{12}$ Y.-H. Liu, M.-C. Xu, S.-S. Yan, and J. Huang, Phys. Status Solidi A 161, 507 (1997).

${ }^{13}$ C. Chappert, K. Le Dang, P. Beauvillain, H. Hurdequint, and D. Renard, Phys. Rev. B 34, 3192 (1986).

${ }^{14}$ S. E. Lofland, S. M. Bhagat, H. L. Ju, C. Xiong, T. Venkatesan, R. L. Greene, and S. Tyagi, Phys. Lett. A 209, 246 (1995).

${ }^{15}$ Yu. V. Goryunov, N. N. Garifyanov, G. G. Khaliullin, I. A. Garifullin, L. G. Tagirov, F. Screiber, Th. Muhge, and H. Zabel, Phys. Rev. B 52, 13450 (1995). 